\title{
Erratum to: CIP2A mediates prostate cancer progression via the c-MYC signaling pathway
}

\author{
Zexiong Guo ${ }^{1} \cdot$ Dehao $^{\mathrm{Liu}^{1}} \cdot \mathrm{Zexuan} \mathrm{Su}^{1}$
}

Published online: 24 July 2015

(C) International Society of Oncology and BioMarkers (ISOBM) 2015

Erratum to:Tumor Biol. 36 (5):3583-3589

DOI 10.1007/s13277-014-2995-5

Tumor Biol. 36(6):4777-4783

DOI 10.1007/s13277-015-3129-4

This article was unintentionally published twice in this journal, by the same authors. Following should be considered the version of record and used for citation purposes: "Zexiong Guo, Dehao Liu, Zexuan Su, CIP2A mediates prostate cancer progression via the c-MYC signaling pathway, Tumor Biology, Volume 36, Issue 6, pages 4777-4783, DOI 10.1007/ s13277-015-3129-4".

The duplicate "Zexiong Guo, Dehao Liu, Zexuan Su, CIP2A mediates prostate cancer progression via the c-MYC signaling pathway, Tumor Biology, Volume 36, Issue 5, pages $3583-3589$, DOI $10.1007 / \mathrm{s} 13277-014-2995-5 "$ is to be ignored.

Torgny Stigbrand/Springer apologizes to the readers of the journal for not detecting the duplication during the publication process.

The online version of the original article can be found at http://dx.doi.org/ 10.1007/s13277-015-3129-4.

The online version of the original article can be found at http://dx.doi.org/ 10.1007/s13277-014-2995-5.

$\mathrm{Zexuan} \mathrm{Su}$

drsuzx6@163.com

1 Department of Urology, First Affiliated Hospital of Jinan University, No. 613 West Huangpu Dadao, Guangzhou 510630, Guangdong,

People's Republic of China 